\title{
Two-photon NAD(P)H and one-photon Flavoprotein autofluorescence imaging to examine the metabolic mechanisms of pancreatic islet beta-cell function
}

\author{
J.V. Rocheleau, * W.S. Head, $*$ and D.W. Piston* \\ * Molecular Physiology and Biophysics, Vanderbilt University, Nashville, TN, 37232-0615
}

The pancreatic islet senses changes in plasma glucose levels through changes in beta-cell redox potential [1]. Previously, we have monitored beta-cell redox potential in isolated pancreatic islets using two-photon NAD(P)H microscopy $[2,3]$. A powerful supplement to $\mathrm{NAD}(\mathrm{P}) \mathrm{H}$ imaging is the simultaneous imaging of flavoprotein autofluorescence. Like $\mathrm{NAD}(\mathrm{P}) \mathrm{H}$, flavin adenine dinucleotide (FAD) and flavin mononucleotide (FMN) show differential fluorescence between their oxidized and reduced states. In contrast to $\mathrm{NAD}(\mathrm{P}) \mathrm{H}, \mathrm{FAD}$ and $\mathrm{FMN}$ are fluorescent in their oxidized state. These molecules are generally quenched when bound as protein cofactors. As a consequence, only two mitochondrial flavoproteins are bright enough to be detected. The first fluorescent flavoprotein source, lipoamide dehydrogenase ( $\mathrm{LipDH})$, is in equilibrium with the mitochondrial NAD(P)H pool (as NAD(P)H increases, LipDH decreases.). The second fluorescent flavoprotein source, electron transfer flavoprotein $(\mathrm{ETF})$, is not in direct equilibrium with mitochondrial $\mathrm{NAD}(\mathrm{P}) \mathrm{H}$. This protein transfers electrons from nine mitochondrial dehydrogenase enzymes to the respiratory chain in eukaryotic cells, and likely contains new information concerning $\beta$-oxidation of fatty acids and electron transport chain activity. Importantly, LipDH fluorescence can be partially separated from unbound FAD/FMN and ETF fluorescence [4]. In particular, the red-shift in the LipDH excitation and emission allows preferential excitation of LipDH at 488 vs. $458 \mathrm{~nm}$ excitation (Fig. 1). We aim to take advantage of this red-shift to establish a more accurate measure of the beta-cell redox potential (NAD(P)H:LipDH ratio).

We examined $\mathrm{NAD}(\mathrm{P}) \mathrm{H}$ and flavin autofluorescence generated from living pancreatic islets using two-photon $(710 \mathrm{~nm})$ and one-photon confocal $(488 \mathrm{~nm})$ microscopy (Fig. 2A \& B). The islets were also imaged after treatment with $3 \mathrm{mM}$ cyanide, which increases cellular redox potential (Fig. 2C \& D). Consistent with an increase in redox potential, NAD(P)H fluorescence increased (Fig. 2A vs. 2C). Also consistent with an increase in redox potential, the $488 \mathrm{~nm}$ excited fluorescence generally decreased. However, there were very bright distinct fluorescent spots (most noticeable in Fig. 2D) that did not decrease in intensity. Interestingly, these regions were also fluorescent during $543 \mathrm{~nm}$ laser excitation, which is inconsistent with flavin fluorescence. Furthermore, these bright spots showed significant co-localization with lysosomal dye and did not significantly overlap with mitochondrial dyes. Therefore, these bright region characteristics are inconsistent with flavin autofluorescence, but consistent with lipofuscin granules. Importantly, to focus our studies on the flavin autofluorescence we need to exclude these lipofuscin regions during image analysis.

References

[1] C.B. Newgard and J.D. McGarry. Annu. Rev. Biochem. (1995) 64:689.

[2] G.H. Patterson et al. Proc. Natl. Acad. Sci. U.S.A. (2000) 97:5203.

[3] J.V. Rocheleau et al. J. Biol. Chem. (2002) 277:30914.

[4] W.S. Kunz. Biochim. Biophys. Acta. (1988) 932:8. 

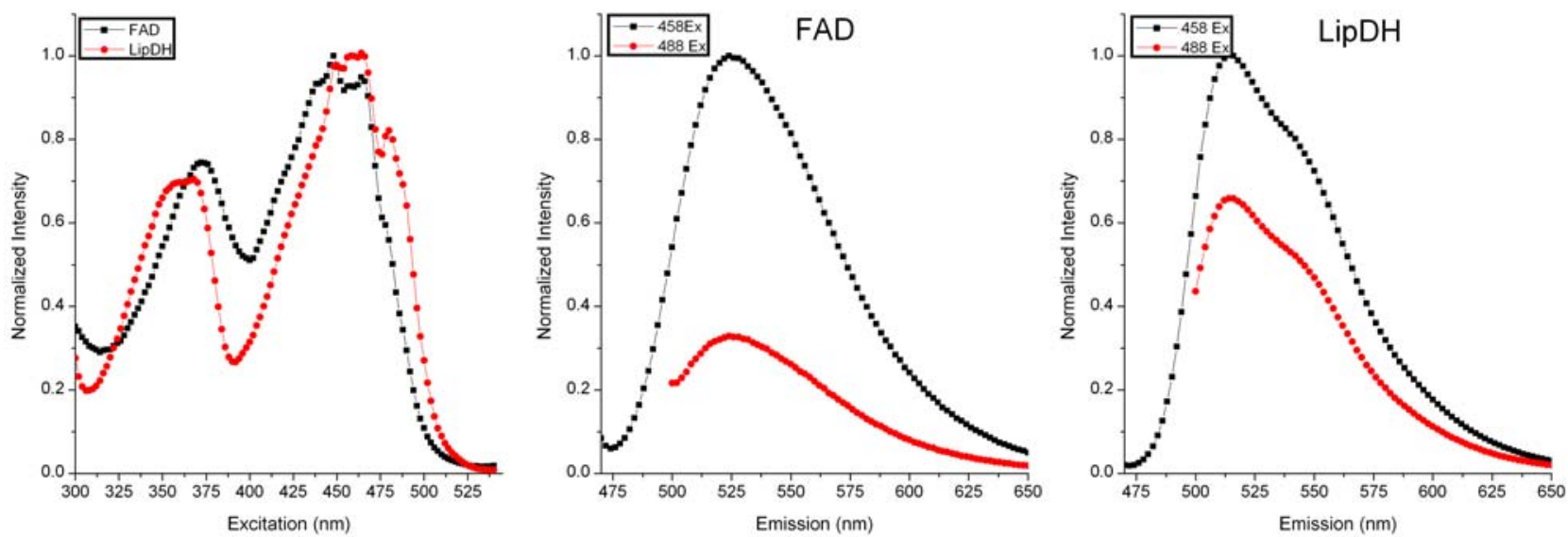

FIG. 1. Excitation and emission spectra of FAD and LipDH (porcine). The excitation spectrum of LipDH is red shifted relative to that of FAD in solution. This causes in an increased fraction of fluorescence observed at 488 vs. $458 \mathrm{~nm}$ excitation for $\mathrm{LipDH}(\sim 0.6)$ than for FAD $(\sim 0.3)$.
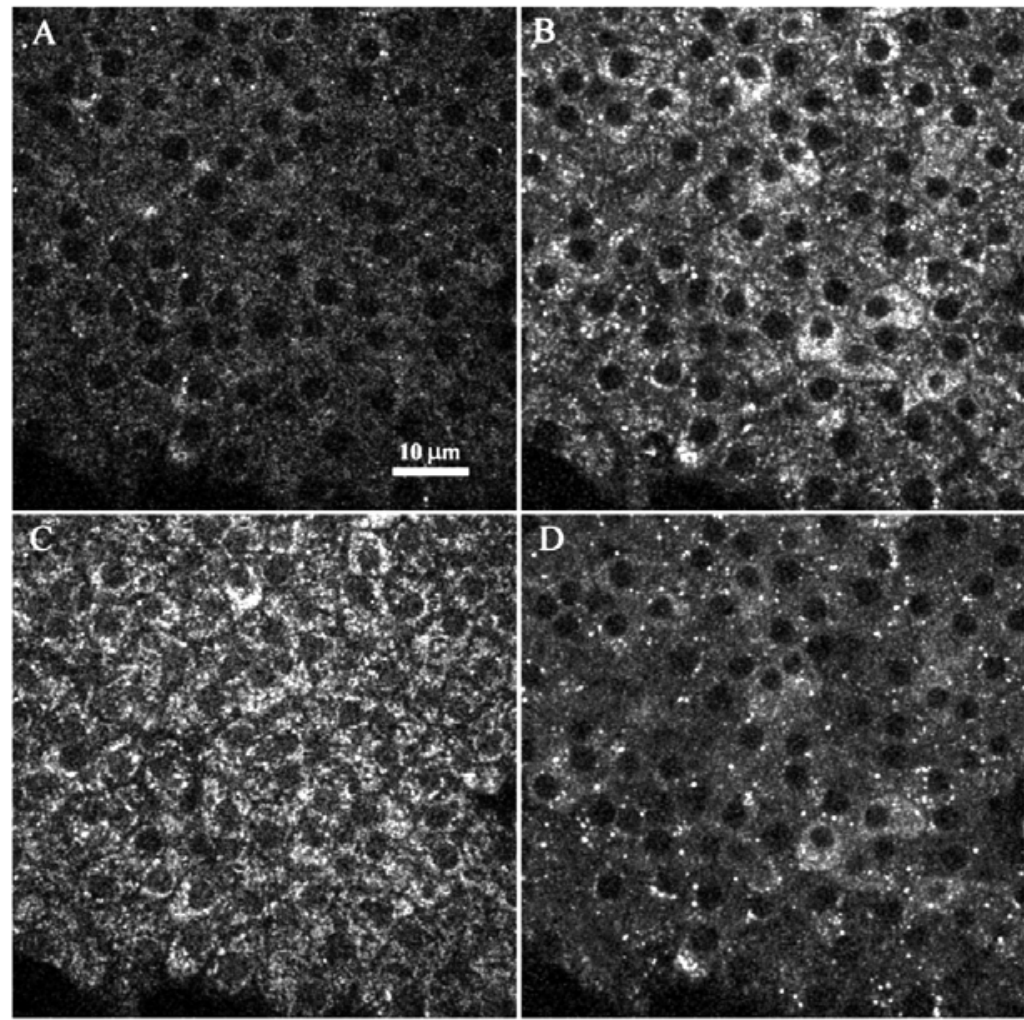

FIG. 2. Images of pancreatic islet autofluorescence. Images were collected using either two-photon excitation at 710nm (A \& C) or using one-photon confocal excitation at $488 \mathrm{~nm}$ (B \& D). The cells were initially in $2 \mathrm{mM}$ glucose which causes a low redox potential in these cells (A \& B). Cyanide $(3 \mathrm{mM})$ was added in order to increase the redox potential of these cells $(C \& D)$. 